\title{
Revitalisierung eines Triftweihers als Wasserfassung und Rentabilitätserhöhung zweier Kleinwasserkraftwerke am Fluss Gail
}

\begin{abstract}
Zusammenfassung: Eine hauptsächlich bis in die 60 -iger Jahre des vergangenen Jahrhunderts zum Holztransport am Gailfluss genutzte Wasserausleitung wurde für eine zusätzliche und teilweise neue Nutzung zur Energieerzeugung adaptiert, saniert und automatisiert. Die Gail transportiert bei höheren Wasserführungen größere Mengen an Grobund Feingeschiebe sowie Eis und diese erfordern für die Wasserfassung bzw. den alten Holztriftweiher besondere konstruktive Voraussetzungen und Lösungen. Durch mehrere umgebaute Geschiebespülschleusen, neue automatische Schützen und Umbauten des Einlaufes, sowie einer neuen Restwasserdotation mit Fischaufstiegshilfe, Abdichtungsmaßnahmen im Weiher und einer Erhöhung des Stauzieles mit einem Umbau des Generators konnten die Betriebsführung verbessert sowie die Energieerzeugung und Leistung der bestehenden Anlagen gesteigert werden.
\end{abstract}

Refurbishment of a logway pond as a power intake and enhancing the rentability of two small hydropower stations on the River Gail

Summary: A diversion channel from the River Gail, which was mainly used for the transport of lumber well into the 1960 s and was also equipped with a small power station, has now been adapted and upgraded for increased electricity generation. The old power station has been extended and a new one built, and the system has been provided with an automatic control system. As the river carries large quantities of coarseand fine-grained bed load as well as ice in times of major flow, the power intake and the old logway pond have called for special design solutions. Several converted bed-load flushing outlets, new automatic gates and conversion of the inlet, as well as provision of a new compensation water system with a fish ladder, waterproofing measures in the pond, a raised reservoir level and conversion of the generator, have improved

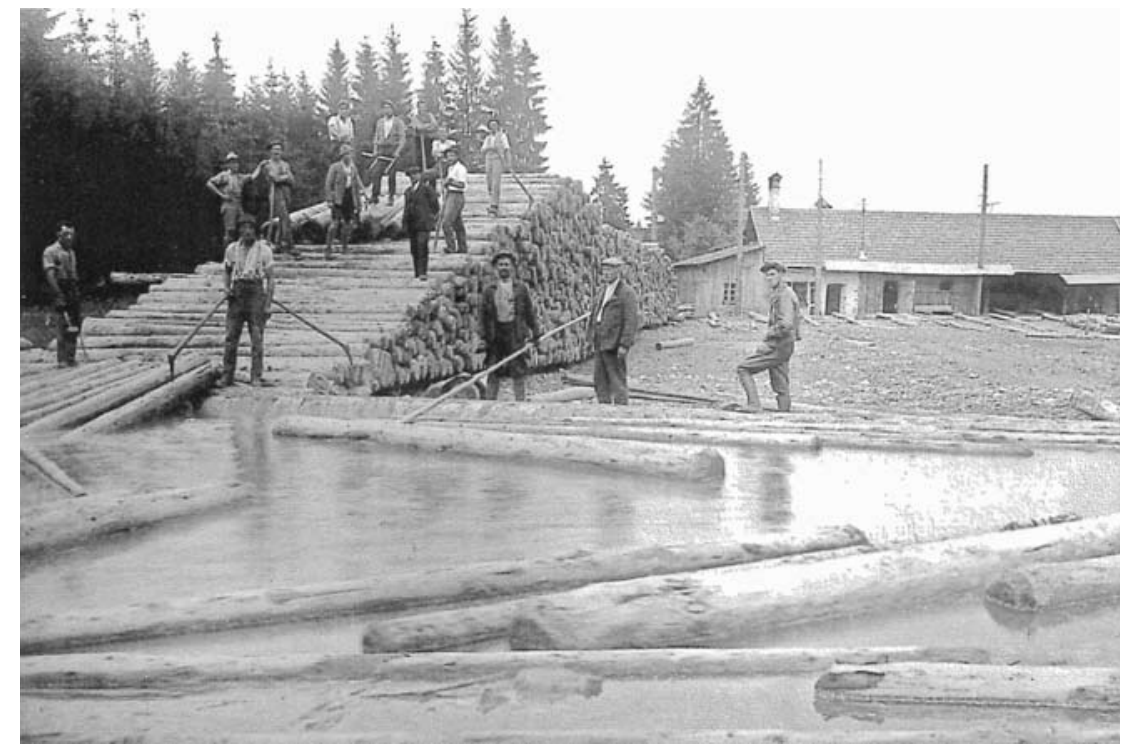

Abb. 1: Holztrift in Wetzmann ${ }^{1}$

plant operation and increased the output and annual generation of the facilities.

\section{Einleitung}

Der Fluss Gail mit seinem Ursprung in Osttirol durchfließt das tief in die südlichen Kalkalpen eingeschnittene Lesachtal und entwässert im Norden die Lienzer Dolomiten sowie die Gailtaler Alpen und im Süden die Karnischen Alpen bevor er kurz vor Kötschach Mauthen in das breiter werdende Obere Gailtal eintritt. Im für lange Zeit schwer zugänglichen Lesachtal spielte Wasser wegen fehlender Straßen eine entscheidende Rolle als Transportmittel für Holz. Am Ausgang des Lesachtales und nach der Gailschlucht wurde das im Fluss getriftete und geflößte Holz im sogenannten Triftweiher Wetzmann angelandet und einer weiteren Verarbeitung im Sägewerk Hasslacher zugeführt. Der Holztrift kam eine besondere wirtschaftliche Bedeutung zu und diese fand in der ersten allgemeinen Regelung der Trift aus dem Jahre 1846 - „Holzschwemm- und
Floßfahrtordnung für den Gailfluss" ihre legistische Verankerung. 1956 wurde schließlich eine Verordnung des Landeshauptmannes von Kärnten erlassen, welche die Trift im Gailfluss auf der Strecke Landesgrenze bis Wetzmann regelte. So wurden von der Wasserrechtsbehörde die Triftzeit, die Entwässerungsstellen, die Holzmenge sowie die Triftbeiträge je Festmeter Holz für Erhaltungsarbeiten an der Gail vorgeschrieben. Die Trift und Flößerei endete de facto mit der Straßenerschließung des Gail- und Lesachtales und die Einstellung erfolgte Mitte der 1960-er Jahre.

Eng verbunden mit der Holztrift war die erste kleine Wasserkraftanlage aus dem Jahre 1923 mit $24 \mathrm{KW}$ und zusätzlichen Transmissionsantrieben. Danach entstand 1927 das noch heute betriebene Kraftwerk Wetzmann I mit einer Fallhöhe etwa $5 \mathrm{~m}$ und einer Leistung von $90 \mathrm{KW}$, welches über den im Seitenschluss des Flusses liegenden Triftweiher das Nutzwasser ausleitete. Triftweiher und Teile der Wehranlage wurden bei einem Hochwasser im November 1951 teilweise zerstört und danach wieder saniert sowie die Verschlüsse erneuert und umgebaut. 


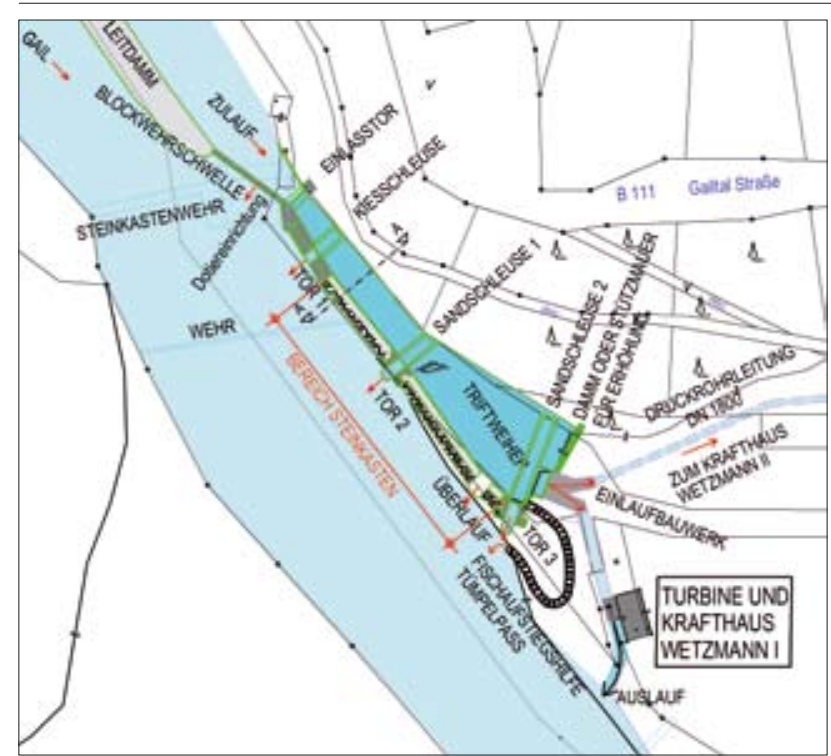

Abb. 2: Lage Triftweihe und Wasserausleitung

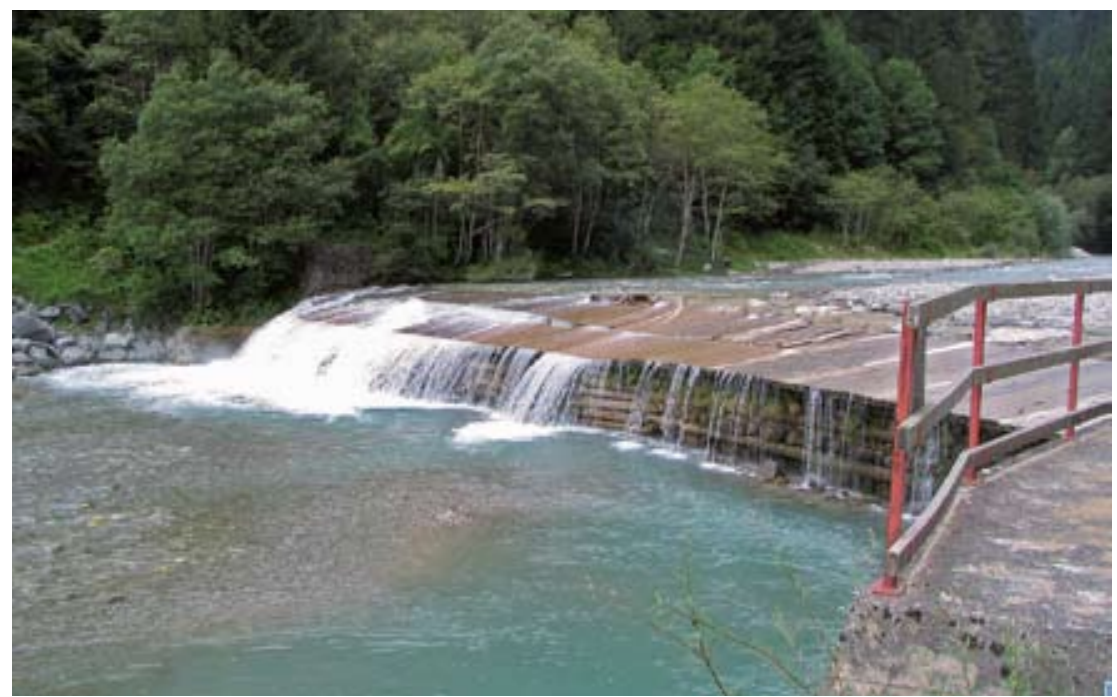

Abb. 3: Holzkastenwehr

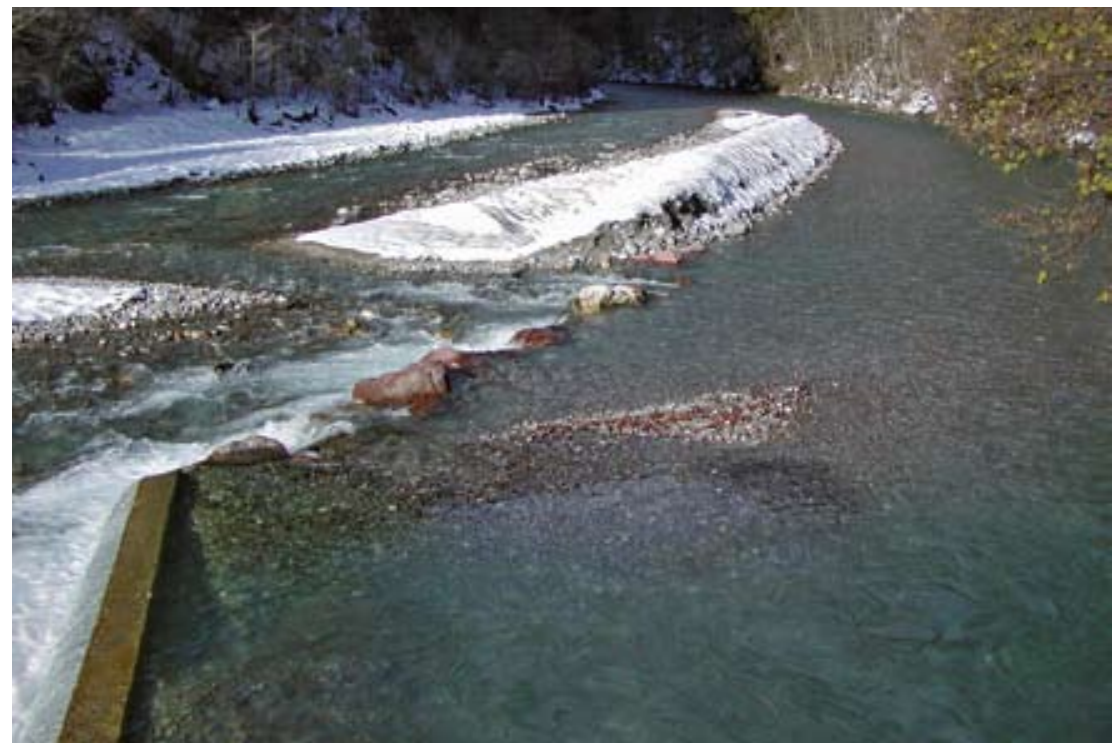

Abb. 4: Oberwasserseitiger Leitdamm

\section{Situierung des Triftweiers}

Nach dem Ende der Holztrift waren durch den bestehenden Weiher ideale Voraussetzungen zur Erweiterung und zum Umbau für eine zusätzliche energetische Nutzung gegeben. Dies führte im Jahre 1988 zur Errichtung eines zweiten Kleinwasserkraftwerkes mit einer Leistung $556 \mathrm{KW}$ und einem Regelarbeitsvermögen von $3,6 \mathrm{GWh}$. Seit dieser Zeit dient der Triftweiher ausschließlich als Wasserfassung, Entsanderanlage und Staubecken für die zwei Kleinwasserkraftwerke Wetzmann I und II. Im Zuge des Baues des Kraftwerkes Wetzmann II wurden der Triftweiher teilweise umgebaut und ein gemeinsames Einlaufbauwerk für die Werke I und II errichtet.

Die Ausleitung der Gail zum Weiher wurde an der Außenkrümmung bzw. am orographisch linken Flussufer der Gail und somit ideal für die Holztrift situiert. Da die Gail in diesem Abschnitt stark Geschiebe führend ist, landete sie jährlich größere Mengen Grobmaterial an, das im Wesentlichen oberhalb der Wehranlage abgelagert und nur bei größeren Hochwässern über die Wehranlage in die nachfolgende Fließstrecke der Gail transportiert wurde. Diese Voraussetzungen waren für einen geordneten Kraftwerksbetrieb teilweise unbefriedigend und führten in der Vergangenheit des Öfteren dazu, dass etwa einmal jährlich an der Außenkrümmung des Flusses eine Rinne ausgebaggert werden musste, damit die ursprünglich vorhandene Tiefenrinne für die Wasserzuführung zum Kraftwerks- bzw. Triftweihereinlauf wieder entstand. Zur weiteren Verbesserung der Betriebsführung wurde wasserrechtlich erwirkt, dass im Gailbett zusätzlich ein Leitdamm aus Flussgeschiebe aufgeschüttet werden konnte, der erfahrungsgemäß etwa jährlich bei mittleren Wasserführungen überströmt und zumindest teilweise wieder abgetragen wird, jedoch bei normalen Wasserführungen die Situation deutlich verbesserte. Weiters kam unmittelbar vor dem Einlauf ein Betonleitsporn zur Ausführung. Ohne Tiefenrinne, Leitdamm aus Flussgeschiebe und Betonleitsporn ist eine Wasserzuführung zum Triftweihereinlauf schwierig und wegen der sich immer verändernden Randbedingungen im Fluss nicht gewährleistet. Zur Sicherstellung der Hochwasserabfuhr wurde der Verlauf des Leitdammes und vor allem seine Höhe bzw. Böschungsneigung behördlich festgelegt und begrenzt. Zufolge dieser Regelung darfnahe des Betonsporns 
die Höhe des Leitdammes diesen um maximal 0,5 $\mathrm{m}$ überragen und die Leitdammoberkante muss flussauf dem Sohlgefälle bzw. dem Wasserspiegelverlauf der Gail folgen. Flussauf darf weiters keine Überhöhung des Dammes erfolgen, sodass eine Überströmung zunächst nahe des Betonsporns erfolgt, der Damm im Ereignisfall dort abgetragen wird und das Gailbett bzw. die Wehranlage zur Hochwasserabfuhr frei gibt. Die Böschungen des Leitdammes müssen flach geneigt, zwischen 1:2 bis 1:5, ausgeführt sein. Aus betrieblichen Gründen war und ist es trotz der Verbesserungsmaßnahmen im Anströmbereich erforderlich, den Einlauf bei mittleren und größeren Hochwässern zu schließen, um vermehrte Eintragungen von Grobgeschiebe und Treibholz etc. in den Triftweiher zu verhindern.

\section{Wehranlage}

Die Wehranlage, ein Holzkastenwehr mit Stein- und Kiesfüllung, wurde in letzter Zeit durch Ausbesserungen einzelner Holzelemente lokal saniert. Im unmittelbaren Unterwasserbereich bildete sich, da ein befestigter Sturzboden nicht vorhanden ist, ein unterschiedlich tiefer Kolk, der zwar periodisch vom Oberwasser mit Geschiebe versorgt wird, jedoch auch periodisch eine Verkippung von Flussbausteinen zur Begrenzung der Kolktiefe erfordert. Gegen eine rückschreitende Eintiefung aus dem Unterwasser wurde die Sohle flussab der Wehranlage durch Stützsohlgurte aus Holzpiloten und Steinsatz gesichert.

\section{Triftweihersanierung}

Im Zuge einer Überprüfung der Standsicherheit und Dichtheit des bestehenden Triftweihers wurde festgestellt, dass Anlagenteile größere Wasserverluste hatten und die Standsicherheit in einigen Bereichen der Stützmauer zwischen dem Triftweiher und dem Gailbett nicht mehr garantiert werden konnten. Weiters waren wichtige Betriebseinrichtungen, wie Spülschützabdichtungen, mechanische Schützenantriebe über Zahnräder und Zahnstangen sowie Holztafelschütze, etc. dringend einer Revision oder Generalsanierung zuzuführen. Die seitliche Steinmauer des Triftweihers war an der Innenseite mit einer Bretterschalung gedichtet und die Sohle teilweise mit einem Holzschussboden abgedeckt. Die Holzkonstruktionen waren teils beschädigt oder zerstört und erfüllten ihre ursprüngliche

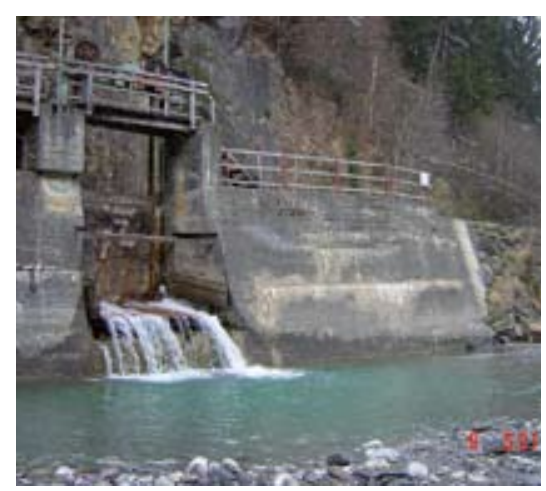

Abb. 5: Undichte Spülschützdichtungen

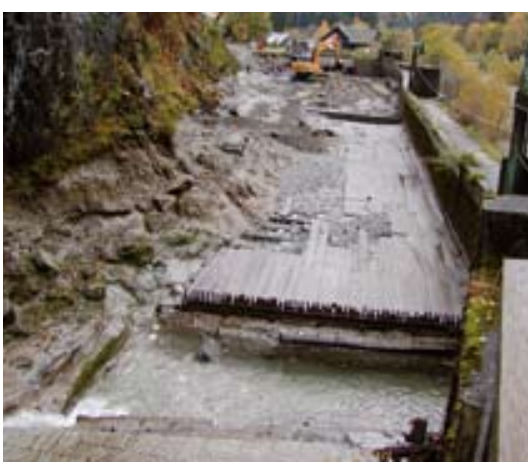

Abb. 7: Zustand Schussboden vor der Sanierung

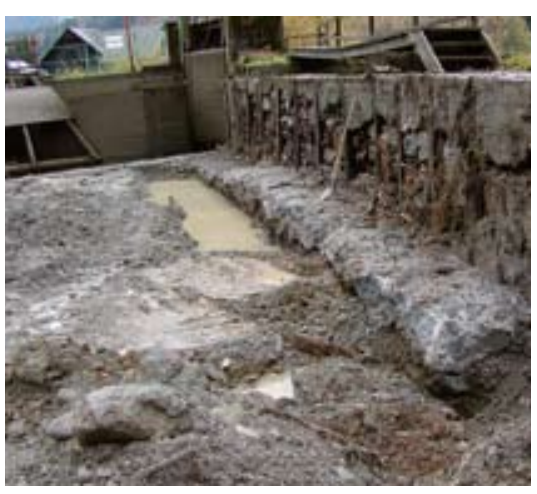

Abb. 6: Zustand des Triftweihers nach Entfernung der Holzauskleidung

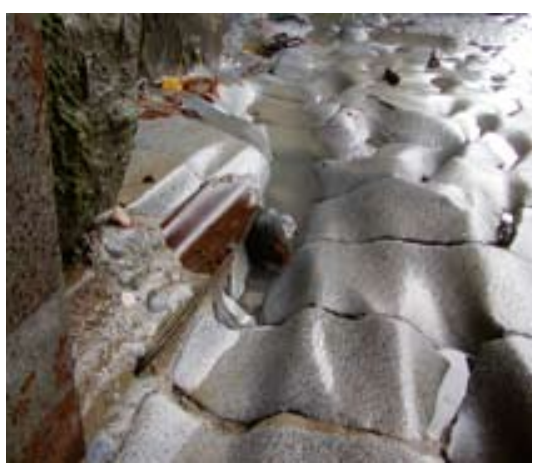

Abb. 8: Geschiebeabrasion im Beton bzw. in Granitauskleidung

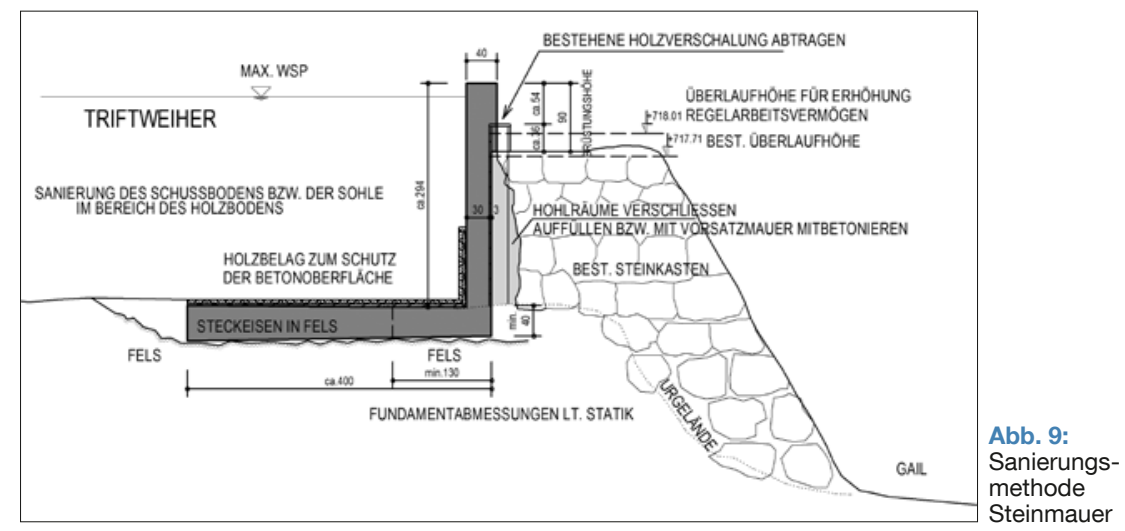

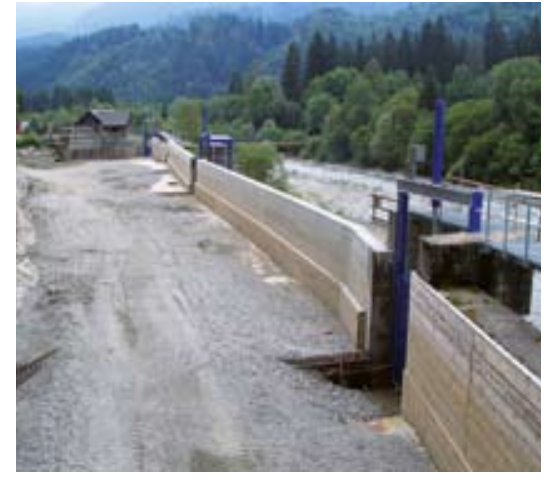

Abb. 10: Neue Weihermauer

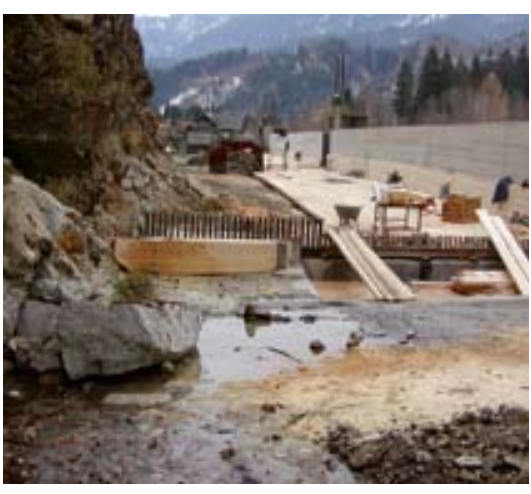

Abb. 11: Neue Weihersohle mit Holzschussboden 


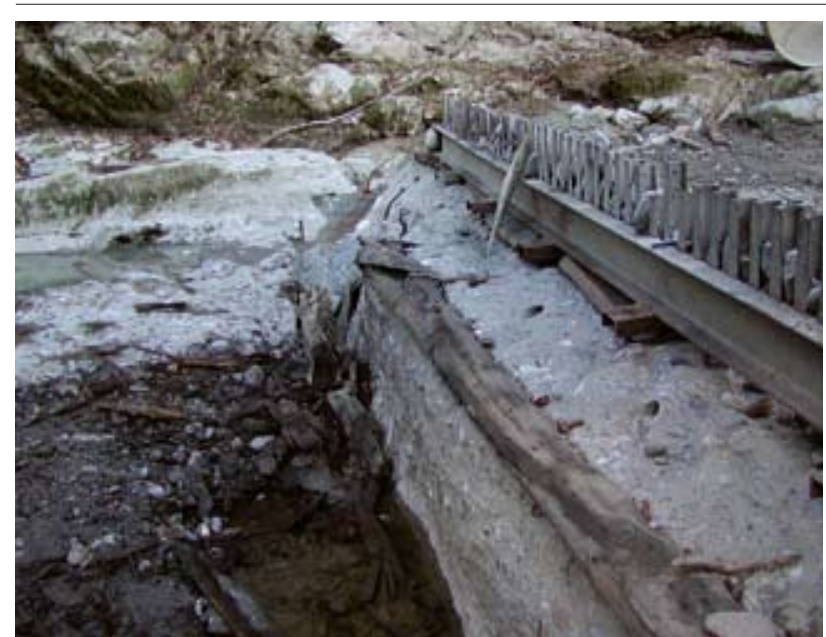

Abb. 12: Beschädigungen der Kiesspülrinne

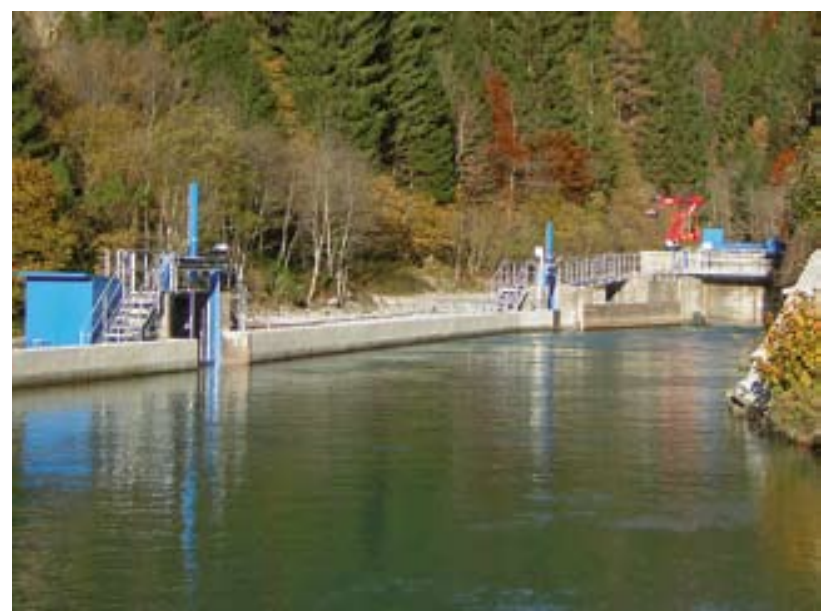

Abb. 14: Triftweiher nach Stauzielerhöhung

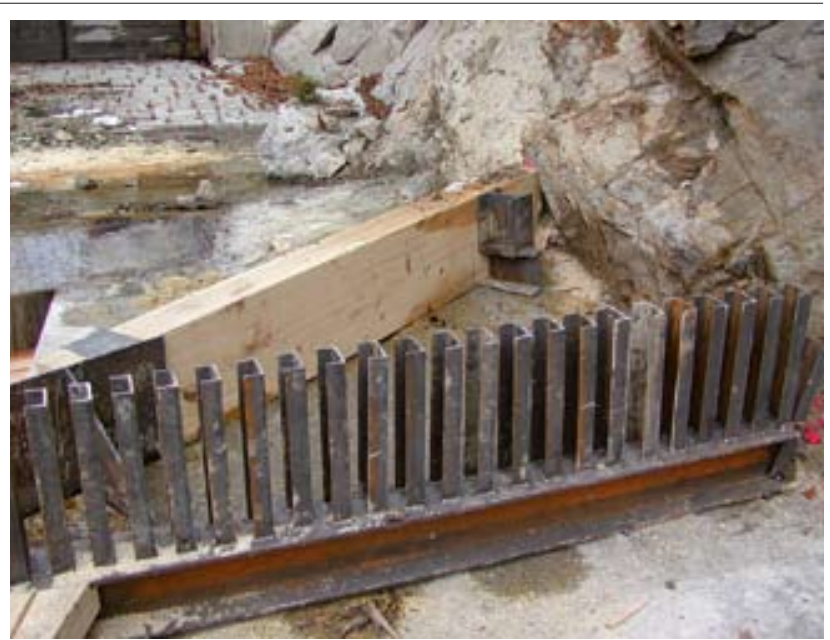

Abb. 13: Erneuerter Kiesfangrechen mit Holzleitbalken

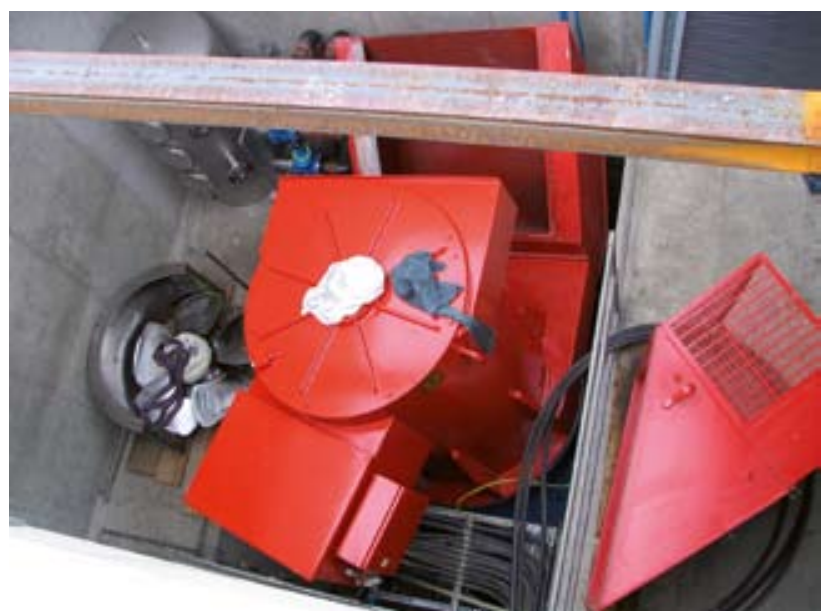

Abb. 15: Einbau des erneuerten Generators
Funktion nicht oder nur mehr eingeschränkt. Weiters wiesen die Beton- und Stahlkonstruktionen im Einlaufbereich und in den Geschiebespülrinnen durch Geschiebeabrasion tiefe Spuren und Schäden auf.

Durch den Eigentümer, die Firma Franz Hasslacher, Säge- Hobel und Elektrowerk, wurde daher im Jahre 2003 entschieden, die Anlage einer Generalsanierung zuzuführen und zusätzlich Geschiebeabfuhrmöglichkeit, Schützenregelungen, Anströmverhältnisse sowie die Rentabilität beider Anlagen durch Erzeugungserhöhung und nach Möglichkeit die Eistriftproblematik im Weiher zu verbessern. Weiters sollte eine von der Behörde im Zuge der Erneuerung des Wasserrechts für Wetzmann I vorgeschriebene Restwasserdotation mit einer Fischaufstiegshilfe umgesetzt werden.

Die bestehende Steinmauer wurde durch eine neue, vorgesetzte Stahlbetonmauer saniert und die offenen Hohlräume in der Steinschlichtung mit Füllbeton aus- gefüllt, so dass ein vollflächiger Verbund zwischen Steinmauer und neuer Wand gewährleistet werden konnte. Da auch die Sicherheit für das Bedienungspersonal erhöht werden sollte, erfolgte dies mit einer $90 \mathrm{~cm}$ hohen Brüstungsmauer, welche ohne besondere Schwierigkeiten auf die neue Weihermauer aufgesetzt werden konnte. In jenen Bereiche der Schussbodensohle des Weihers, die mit Bretterschalungen abgedichtet waren, wurde eine neue Stahlbetonplatte ausgeführt. Gailseitig mussten am luftseitigen Steinmauerfuß weiters größere Hohlräume verschlossen und die Steinschlichtung zur Flusssohlsicherung stabilisiert werden.

Die Kiesspülrinne in der Weihersohle, die am stärksten durch die häufige Abrasionsbeanspruchung in Mitleidenschaft gezogen war, wurde komplett erneuert, zusätzlich mit einer Stahlpanzerung verstärkt sowie mit einem neuen Kiesfangrechen versehen. Die beiden nachgeschalteten Sandfang- und Sandspülrinnen wurden neu betoniert, die Rin- nenkanten stahlgepanzert und die Sohlen mit einem Lärchenbelag abgedeckt. Alle Führungseinrichtungen der drei Verschlüsse und das alte Übereich des Weihers waren ebenfalls zu erneuern.

\section{Erhöhung von Leistung und Energieerzeugung}

Zur Erhöhung der Energieerzeugung und Verbesserung der Anströmverhältnisse zu den Triebwassereinläufen beider Kraftwerke wurden mehrere Varianten untersucht und daraus ergab sich, dass eine Stauzielerhöhung wirtschaftlich sowie technisch sinnvoll ist und umgesetzt werden kann. Untersuchungen und Nachweise für das Kraftwerk Wetzmann II erbrachten, dass der vorhandene Generator den Engpass für die Anlage und somit die Vergrößerung der Fallhöhe und des Ausbaudurchflusses darstellte. Die Rohrturbine war durch die elektrotechnischen Leistungsgrenzen der Anlage auf eine maximale Turbinenleistung an der Genera- 


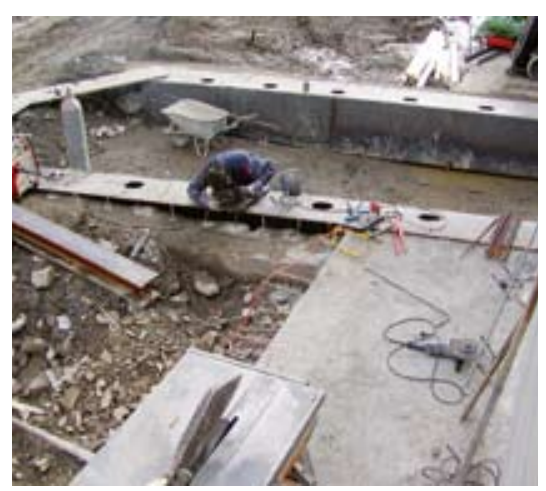

Abb. 16: Stahlpanzerung der Spülgassen

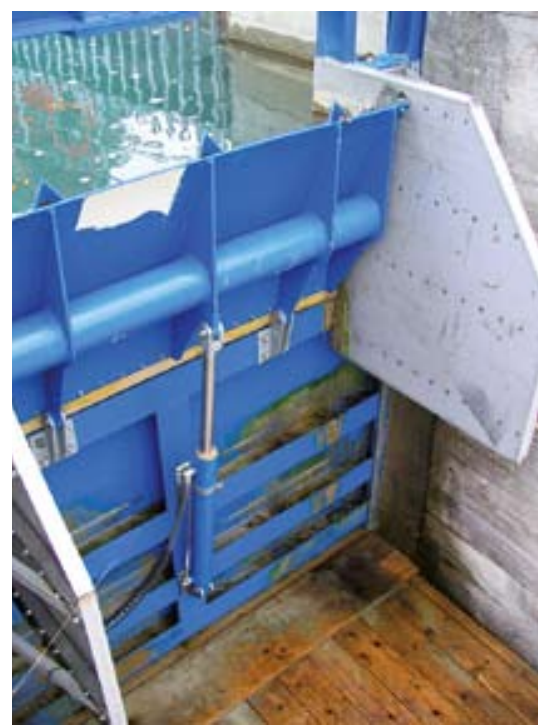

Abb. 19: Spülschütz mit Überlaufklappe

torwelle von $556 \mathrm{KW}$ gedrosselt und konnte laut Angaben der Herstellerfirma Andritz auf eine Dauerbetriebsleistung von $621 \mathrm{KW}$ erhöht werden. Das Getriebe verfügte ebenfalls noch über Reserven zur geplanten Kapazitätserweiterung und im Zuge der Sanierung der Anlage waren daher für Turbine und Getriebe lediglich reguläre Servicearbeiten erforderlich.

Der Umbau des Generators vom vorhandenen Leistungsengpass bei $700 \mathrm{kVA}$ auf die neue, geforderte Leistung von $850 \mathrm{kVA}$ umfasste den Austausch der Generatorlager, den Einbau eines einstellbaren $\cos \varphi$-Reglers, die Verstärkung von Generatorsammelschienen und Generatorausleitungen, die Verstärkung der Verkabelung bis zum Blocktransformator sowie den Einbau eines stärkeren Synchronisierschalters.

Mit diesen Maßnahmen wurde eine optimale Ausnutzung der elektrischen und maschinellen Anlagenteile bei vorgesehener Stauzielerhöhung um $30 \mathrm{~cm}$ und Erhöhung des Ausbaudurchflusses Wetz-

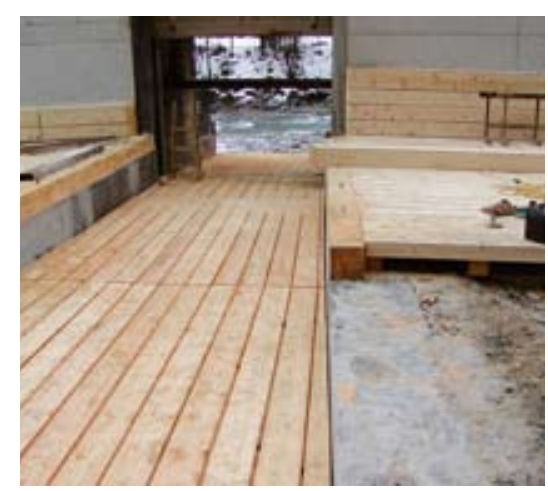

Abb. 17: Lärchenholzabdeckung der Spülgassen

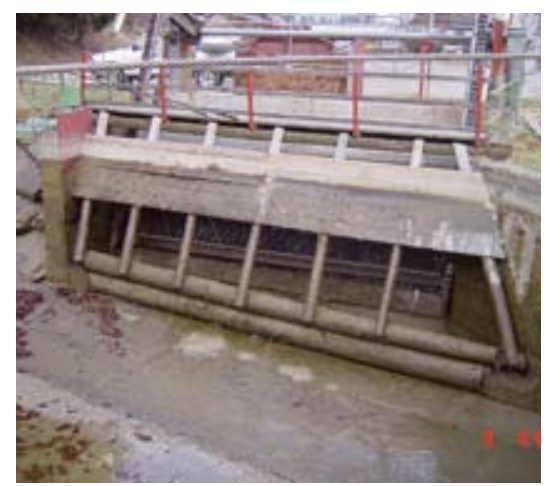

Abb. 20: Alter Triebwassereinlauf mit Holzstaubalken

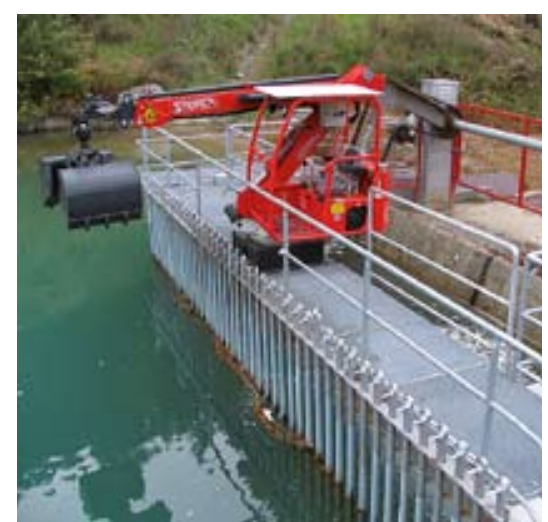

Abb. 22: Grobrechen mit Kleinbagger

mann II von bisher genehmigten $5,0 \mathrm{~m}^{3} / \mathrm{s}$ auf $6,0 \mathrm{~m}^{3} / \mathrm{s}$ möglich. Nach behördlicher Genehmigung der Änderungen konnte auch der Nachweis gemäß $\S 3$ Absatz 2 des Ökostromgesetzes über eine Erhöhung des Regelarbeitsvermögens um mindestens $15 \%$ erbracht und damit der erhöhte Stromtarif für die Einlieferung erreicht werden. Bezogen auf die durchschnittliche Erzeugung der vorangegangenen Jahre steigerte sich das Regelarbeitsvermögen um etwa $20 \%$ auf rund $4,4 \mathrm{GWh}$. Im Zuge der Revitalisierung wurde auch beim Kraftwerk Wetzmann I eine Kon-

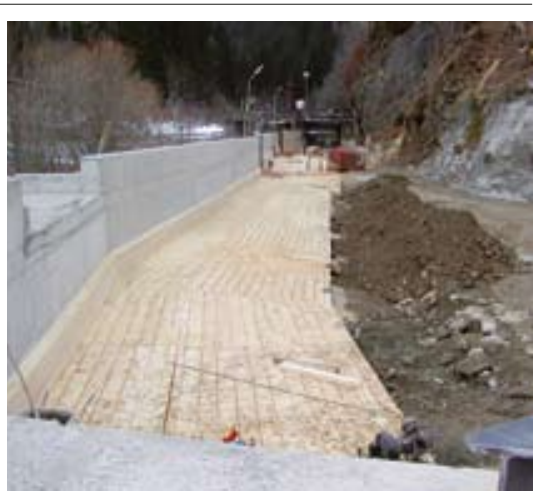

Abb. 18: Triftweihersohle mit Lärchenholzschussboden

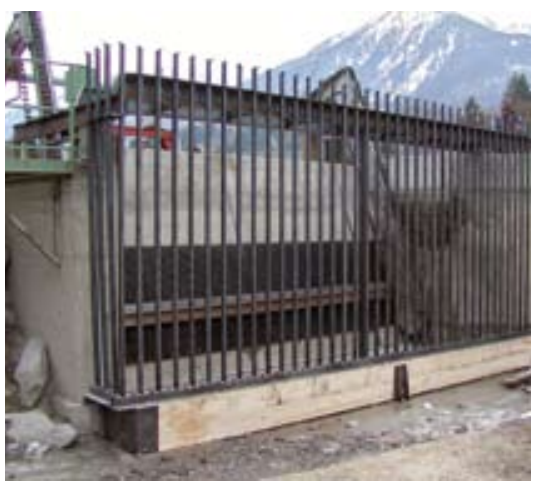

Abb. 21: Neue Grobrechenkonstruktion mit ziehbaren Stäben

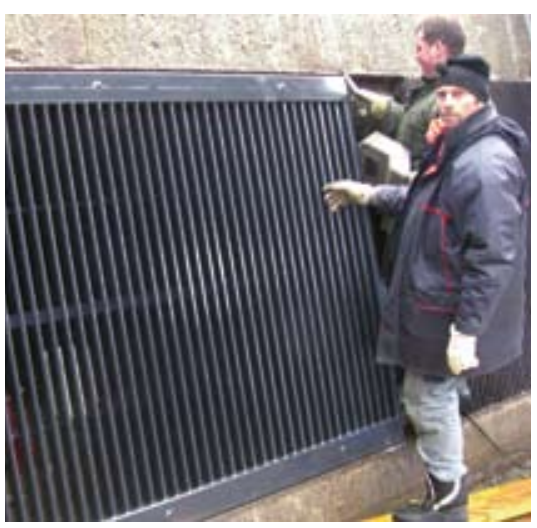

Abb. 23: Feinrechen mit Beheizung aus Biomasseheizanlage

senswassermengenerhöhung von 2,5 auf $2,8 \mathrm{~m}^{3} /$ s erwirkt.

\section{Kies- Und Sandspülschleusen}

Durch die am Gailfluss gegebenen Randbedingungen eines ausgeprägten Geschiebetransportes und somit eines möglichen Eintrages von Grob- und Feinmaterial in den Triftweiher kommt einer gut funktionierenden Geschiebeentlandung besondere Bedeutung zu. Das in den 1950er Jahren entwickelte und umgesetzte Konzept mit drei nach einander angeordneten 


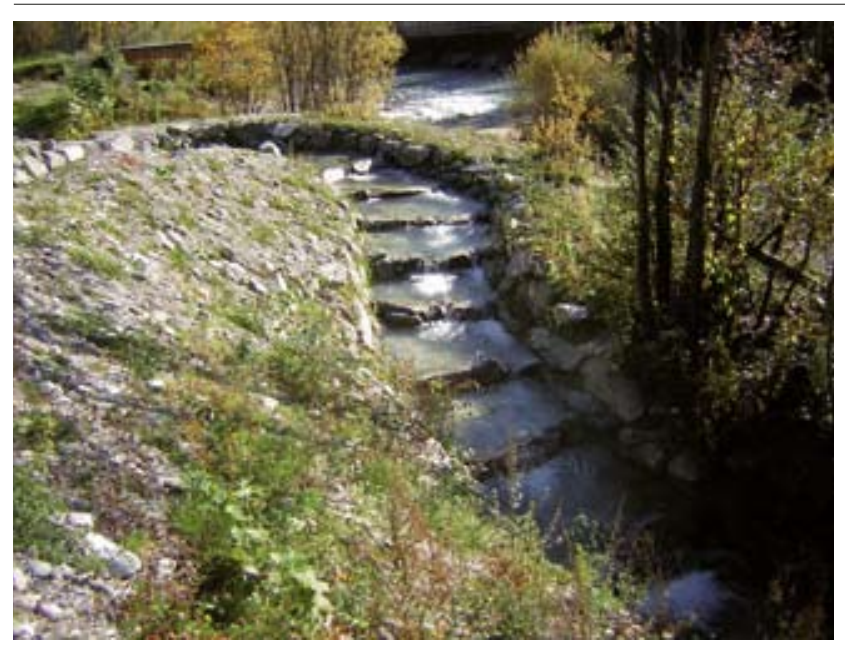

Abb. 24: Fischaufstiegshilfe als Tümpelpass

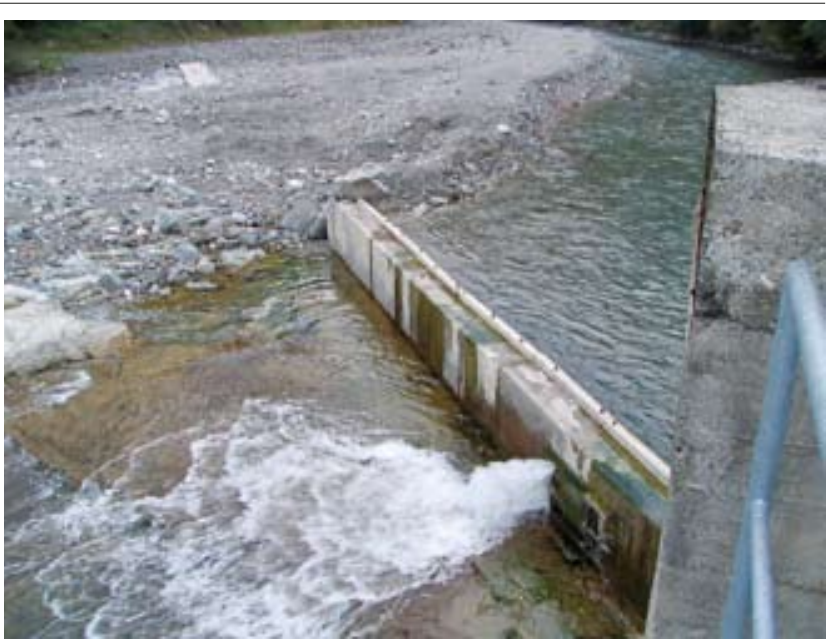

Abb. 25: Restwasserdotation beim Triftweihereinlauf
Spülrinnen in der Weihersohle hat sich grundsätzlich gut bewährt und wurde deshalb auch beibehalten. Vor allem die erste Spülschleuse, die für die Entlandung von Kies dient, war stark angegriffen und beschädigt. Es wurde die gesamte Rinne neu betoniert und hoch beanspruchte Bauteile stahlgepanzert, ein neuer Geschieberechen mit Leitbalken eingebaut sowie die Sohle der Spülschleuse mit Lärchenholzbohlen belegt. Für die beiden anderen Spülschleusen, vor allem zur Entlandung Sand vorgesehen, kam ein entsprechendes Revitalisierungskonzept zur Anwendung. Auf der gesamten neuen Stahlbetonsohle des Weihers wurde ebenso wie an den unteren Stahlbetonwänden ein Lärchenbohlenbelag aufgebracht. Dieser ist erfahrungsgemäß wesentlich widerstandsfähiger gegen Fein- und Grobgeschiebe als herkömmlicher Beton. Ein Einsatz von Spezialbeton kann aus Kostengründen nicht in Frage.

Die vorhandenen, alten Spülschützentafeln aus Holzbohlen mit einfassendem Stahlrahmen waren grundsätzlich noch in einem relativ Zustand, die Dichtungen allerdings teilweise stark beschädigt und die Zahnstangen bzw. die Zahnräder bereits stark abgenutzt. Die Führungsschienen zeigten vor allem bei der Kiesspülschleuse stärkere Schäden und wären nur noch aufwändig zu reparieren gewesen. Daher entschied sich der Betreiber alle drei alten Tafelverschlüsse durch neue Gleitschützen aus Stahl mit beheizbaren Dichtungskonstruktionen zu ersetzen und die Führungen in Nirostahl auszuführen. Um Schwemmgut leichter abführen zu können wurde das Spülschütz vor dem Triebwassereinlauf mit einer hydraulischen Überlaufklappe konzipiert. Alle drei Schützen bekamen hydraulische Hubeinrichtungen mit modernen Steuerungen.

Der vorhandene Feinrechen beim Triebwassereinlauf wurde ebenfalls generalsaniert und zusätzlich eine Hälfte mit beheizbaren Fischflossenstäben ausgerüstet. Die Beheizung erfolgt durch Wärme aus der eigenen Biomasseheizanlage und soll ein Vereisen der Rechenstäbe verhindern. Anstelle des vorhandenen, vorgesetzten Holzstaubalkens kam eine neue Grobrechenkonstruktion mit im Winter ziehbaren Rechenstäben und Bedienungssteg zur Ausführung. Die vorhandene Rechenreinigungsmaschine und ein kleiner Greiferbagger mussten nicht angepasst und verändert werden.

\section{Fischaufstiegshilfe und Restwasserabgabe}

Zur Erfüllung der Wasserrahmenrichtlinie und um einen durchgehenden Fließgewässercharakter der Gail in der kurzen Ausleitungsstrecke aufrecht zu erhalten sowie die Fischwanderung zu ermöglichen wurde behördlicherseits eine dauernd und kontrollierbar abzugebende Pflichtwassermenge von $7401 / \mathrm{s}$ vorgeschrieben. Diese muss durch Abgabe von 2501/s über eine neu zu errichtende Fischaufstiegshilfe und von 4901/s im Bereich der Ausleitungsstrecke bzw. durch gleichmäßig verteilte Dotationen über eine Öffnung vor dem Triftweihereinlauf und weitere regelbare Öffnungen in den Spülschützen erfolgen.

Für die neue Fischaufstiegshilfe kam ein Tümpelpass mit speziell gestal- teten und konstruierten Zu- bzw. Austritten zur Ausführung. Die Hinzuziehung eines Fischökologen für Entwurf- und Detailgestaltungsvorschläge war sehr hilfreich.

\section{Zusammenfassung}

Die Revitalisierungsarbeiten an den Kraftwerken bzw. die Neuerrichtung der Fischaufstiegshilfe erfolgte 2003 sowie 2004. Die Gesamtbaumaßnahmen konnten endgültig im Sommer 2005 fertig gestellt werden. Die Anlagen haben sich seit der Wiederinbetriebnahme gut bewährt. Die Gesamtkosten der Revitalisierung betrugen etwa $€ 600.000$,-

Korrespondenz:

Univ. Prof. Dipl. Ing. Dr. techn. Peter Tschernutter Institut für Wasserbau und Ingenieurhydrologie Karlsplatz 13 1040 Wien

E-Mail: peter.tschernutter@kw.tuwien.ac.at

Franz-Christian Berger

Geschäftsführender Gesellschafter Hasslacher Holzwerke Ges.m.b.H

9640 Kötschach 132

E-Mail: meisterfenster@hasslacher.net

Ing. Michael Adunka

Ingenieurbüro Tschernutter

Fabriksteig 10/1

9500 Villach

E-Mail: office@zt-tschernutter.at

\section{LITERATUR}

1125 Jahre Gailregulierung, Amt der Kärntner Landesregierung, Abteilung 18-Wasserwirtschaft, 9010 Klagenfurt, Bundesministerium für Land- und Forstwirtschaft, Umwelt und Wasserwirtschaft, 1012 Wien, Hermagor, 2001 